\title{
INTERFERENCE BETWEEN OUTBREAKS OF RESPIRATORY VIRUSES
}

\author{
G Ånestad (gabriel.anestad@fhi.no)1, S A Nordbø²,3 \\ 1. Department of Virology, Division of Infectious Disease Control, Norwegian Institute of Public Health, Oslo, Norway \\ 2. Department of Medical Microbiology, St.Olavs Hospital, Trondheim University Hospital, Trondheim, Norway \\ 3. Institute of Laboratory Medicine, Children's and Women's Health, Norwegian University of Science and Technology, \\ Trondheim, Norway
}

This article was published on 15 October 2009.

Citation style for this article: Ånestad G, Nordbø SA. Interference between outbreaks of respiratory viruses. Euro Surveill. 2009;14(41):pii=19359. Available online: http:// www.eurosurveillance.org/ViewArticle.aspx?ArticleId $=19359$

To the editor: Norway, like several other European countries, has experienced a delay in the expected outbreaks with pandemic H1N1 influenza. In a recent paper from Sweden it has been postulated that this delay, at least partly, was caused by interference with other respiratory viruses. This view is supported by the fact that a relatively high rhinovirus activity was registered in late summer and early autumn in Sweden [1].

St. Olav's University Hospital in Trondheim, Norway has for several years conducted extensive laboratory surveillance of respiratory viruses including rhinoviruses. The Figure shows the rhinovirus infections diagnosed in Trondheim in the past three years. An increase in diagnosed rhinovirus infections was observed during late summer and early autumn in 2007 and during autumn 2009.

Compared with the complex and enveloped influenza virus particle, rhinoviruses may have advantages at times of the year when the climatic conditions are suboptimal for respiratory viruses. Thus, if the interference theory is correct, rhinoviruses will usually not have any competition with other respiratory viruses during late summer and early autumn, and the interference effect will be obscured. On the other hand, if a competing virus is introduced,

\section{F I G U R E}

Laboratory-confirmed rhinovirus infections, January 2007-September 2009, Trondheim, Norway $(n=646)^{*}$

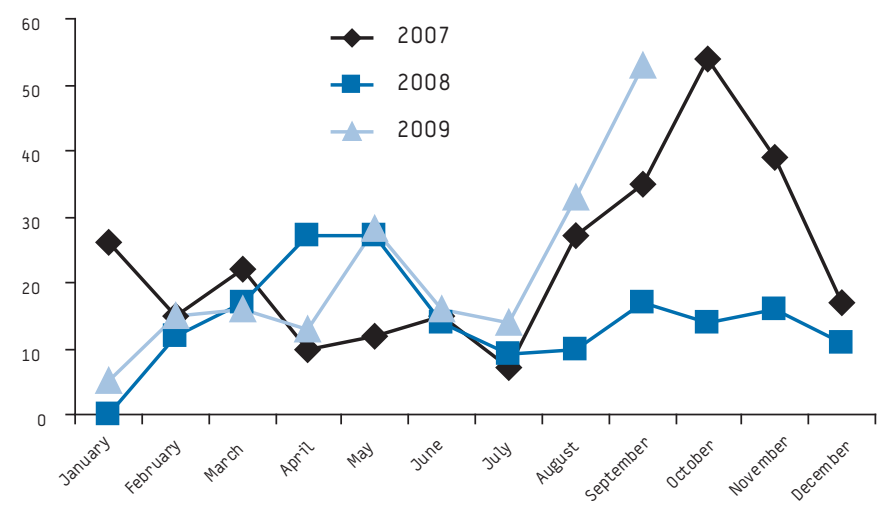

the interference activity will be apparent in a delayed outbreak development. As an illustration of this, pandemic H1N1 influenza virus was first diagnosed at St. Olav's Hospial in May 2009, and although a little peak in influenza cases was observed near the end of July 2009 , only $5-10 \%$ of specimens from patients with influenza-like illness have tested positive for pandemic H1N1 influenza virus. The great majority of these patients were infected with rhinoviruses and to a lesser extent with parechovirus.

Greer et al. observed that co-infections with rhinoviruses and other respiratory viruses were more uncommon than expected, indicating that rhinovirus infection may render the host less likely to be infected with other viruses [2].*

Based on observations in Norway, epidemiological interference between several epidemic viruses including influenza virus has been suggested [3-5]. The present observations may lend some further support to this hypothesis.

*Author's correction: On request of the authors, the number of rhinovirus infections in September 2009 was corrected in the figure on 21 0ctober 2009, and one sentence was added introducing a new reference.

\section{References}

1. Linde A, Rotzén-Östlund M, Zweygberg-Wirgart B, Rubinova S, Brytting M. Does viral inteference affect spread of influenza? Eurosurveillance Eurosurveill. 2009;14(40):. pii=19354. Available from: http://www.eurosurveillance.org/ ViewArticle.aspx?ArticleId $=19354$

2. Greer RM, McErlean P, Arden KE, Faux CE, Nitsche A, Lambert SB, et al. Do rhinoviruses reduce the probability of viral co-detection during acute respiratory tract infections? J Clin Virol. 2009;45(1):10-5.

3. Anestad G. Interference between outbreaks of respiratory syncytial virus and influenza virus infection. Lancet. 1982;1(8270):502.

4. Anestad G. Surveillance of respiratory viral infections by rapid immunofluorescence diagnosis, with emphasis on virus interference. Epidemiol Infect. 1987;99(2):523-31.

5. Anestad G, Vainio K, Hungnes O. Interference between outbreaks of epidemic viruses: additional Norwegian observations. Scand J Infect Dis. 2009;41(5):381-2. 